\title{
Germanica
}

\section{Les relations entre la Garde de fer et l'Allemagne,}

1919-1938

Die Beziehungen zwischen der rumänischen Eisernen Garde und Deutschland, 1919-1938

The relations between the Romanian Iron Guard and Germany, 1919-1938

\section{Traian Sandu}

\section{(2) OpenEdition}

\section{Journals}

Édition électronique

URL : http://journals.openedition.org/germanica/4312

DOI : $10.4000 /$ germanica.4312

ISSN : 2107-0784

Éditeur

Université de Lille

Édition imprimée

Date de publication : 31 juillet 2018

Pagination : 33-55

ISBN : 9782913857414

ISSN : 0984-2632

Référence électronique

Traian Sandu, «Les relations entre la Garde de fer et l'Allemagne, 1919-1938 », Germanica [En ligne], 62 | 2018, mis en ligne le 31 juillet 2020, consulté le 15 janvier 2021. URL : http://

journals.openedition.org/germanica/4312 ; DOI : https://doi.org/10.4000/germanica.4312

(c) Tous droits réservés 


\title{
Les relations entre la Garde de fer et l'Allemagne, 1919-1938
}

\author{
Traian SANDU \\ Université Sorbonne Nouvelle - Paris 3
}

\begin{abstract}
À première vue, l'approche du phénomène fasciste par les relations internationales n'est pas celle privilégiée par les érudits, pour de nombreuses raisons, mais principalement parce que leurs efforts dans le cas de soi-disant «petits fascismes » - en fait ce que j'appelle de grands fascismes dans de petits pays ${ }^{1}$ - ont consisté à démontrer avant tout l'autonomie des fascismes locaux par rapport aux mouvements ou aux régimes fascistes des grandes puissances italienne et allemande.

Cette approche légitime incite d'abord à une méthodologie comparative, et non à une relation, afin de construire une définition du phénomène à partir de ses multiples manifestations nationales, puis de subsumer les mouvements et les régimes à la définition pour contrôler s'ils sont adaptés ou non. Une telle méthode est certainement indispensable pour proposer un discours général sur le fascisme et quitter les approches nationales idiosyncrasiques. Toute la littérature visant à
\end{abstract}

1. - Traian Sandu, « Conclusion : Un fascisme centre-européen harmonique au sein du new consensus, et au-delà : la "nouvelle vague" », dans Traian Sandu (dir.), Vers un profil convergent des fascismes? "Nouveau consensus » et religion politique en Europe centrale, Paris, L'Harmattan, coll. «Cahiers de la Nouvelle Europe », 2010, p. 261-271.

GERMANICA, 2018, LXII, pp. 33 à 55. 
dégager un "idéal-type » wébérien d'un fascisme générique se fonde sur le comparatisme ${ }^{2}$.

Néanmoins, elle pourrait aussi aboutir à une argumentation circulaire - définissant comme fascistes les mouvements, les régimes et les idéologies d'abord mobilisés pour la définition - si le va-et-vient entre la définition théorique du modèle et les cas nationaux appuyés sur la recherche archivistique n'est pas correctement ajusté. Ainsi les approches relationnelles et de transfert s'avèreront-elles certainement utiles pour évaluer certaines conclusions comparatives dans l'optique des acteurs euxmêmes et dans la manière dont ils ont considéré ces points communs idéologiques et structurels et leurs relations. Ces approches génèreront aussi quelques distorsions, comme la surreprésentation des élites.

Le point principal de l'argumentation est la prééminence de l'idéologie sur la Realpolitik dans les relations internationales et donc de la spécificité du fascisme sur les autres mouvements et régimes nationalistes et antisémites dans les deux phases, avant et après la prise du pouvoir. Pour nous, les États fascistes ne sont pas des États westphaliens, ils n'évoluent pas dans le soi-disant « désordre international », mais plutôt dans un système géopolitique axé sur l'idéologie. Par conséquent, nous pouvons analyser les relations internationales multi-scalaires des fascistes roumains de la Garde de fer ${ }^{3}$ à travers une étude des transferts

2. - George Mosse, La Révolution fasciste, Paris, Seuil, 2003 ; Stanley G. Payne, A History of Fascism: 1914-1945, Madison, The Wisconsin University Press, 1995 ; Roger Eatwell, Fascism, Londres, Chatto \& Windus, 1996 (nouvelle édition : Fascism. A History, Pimlico, 2003) et Roger Griffin, The Nature of Fascism, Londres, Pinter, 1991 ; Emilio Gentile, Qu'est-ce que le fascisme? Histoire et interprétation, Paris, Gallimard, 2004.

3. - Traian Sandu, Un Fascisme roumain. L'histoire de la Garde de fer, Paris, Perrin, 2014 ; Armin Heinen, Die Legion „Erzengel Michael“ in Rumänien: soziale Bewegung und politische Organisation. Ein Beitrag zum Problem des internationalen Faschismus, Munich, Oldenbourg-Verlag, 1986 (nous utilisons la traduction roumaine : Legiunea "Arhanghelul Mihai”. Mişcare socială şi organizaţie politică. O contribuţie la problema fascismului international, Bucarest, Editura Humanitas, 1999) ; Constantin Iordachi, Charisma, Politics and Violence : The Legion of the "Archangel Michael » in Inter-war Romania, Trondheim Studies on East European Cultures \& Societies, décembre 2004 ; Mihai Chioveanu, Feţele fascismului. Politică, ideologie şi scrisul istoric în secolul $X X$ [Les visages du fascisme. Politique, idéologie et écriture historique au XXe siècle], Bucarest, Ed. Universităţii Bucureşti, 2005. Plusieurs autres auteurs ont fourni de plus brefs essais, voir par exemple : Valentin Săndulescu, « On the ideological characteristics of the Romanian Legionary Movement : a synthetic account », dans Studia Universitatis Petru Maior, Series Historia, 2005, p. 141-154 ; Traian Sandu, « De l'antisémitisme au fascisme en Roumanie : naissance du Roumain nouveau régénéré par la révolution de droite », dans Analele Universităţii Bucureşti, Année X, 2008, p. 32-46. Pour l'idéologie légionnaire, voir aussi Constantin Iordachi, « God's chosen warriors. Romantic Palingenesis, militarism and fascism in modern Romania », dans Constantin Iordachi, Comparative Fascist Studies. New Perspectives, Londres, Routledge, coll. « Rewriting Histories », p. 316-357. Pour la période ultérieure à 1938, voir Andreas 
sociaux et pas seulement à travers une approche empirique fondée sur les relations internationales entre les États libres.

Je propose une présentation chronologique, mais qui joue sur trois niveaux :

- le transfert simple par contact direct avec d'autres milieux allemands apparentés - essentiellement le Parti national-socialiste et ses officines ;

- le rôle de liaison active des personnalités légionnaires comme intermédiaire dans le rapprochement entre nationaux-socialistes allemands et fascistes italiens, grâce à leur intégration dans le réseau européen d'extrême droite au milieu des années trente, toujours une période de tensions et de compétition entre les deux puissances fascistes ;

- l'interférence de l'idéologie fasciste dans les relations à plusieurs niveaux entre les États, les partis et les individus, jouant un rôle dramatique lors des crises internationales.

La comparaison des idéologies n'est donc pas l'objet de cet article. Elle apparaîtra occasionnellement, si nécessaire.

\section{Berlin, modèle pour le fondateur des légionnaires de l'Archange Michel}

Le premier niveau intégrant les leaders préfascistes au réseau nationaliste radical européen est le simple transfert de compétences idéologiques et de mobilisation. J'ai abordé ailleurs les transferts intellectuels croisés de l'Action française et du fascisme italien sur les doctrinaires légionnaires ${ }^{4}$, je me concentrerai ici plutôt sur des contacts concrets, et sur la principale personnalité légionnaire, Corneliu Zelea Codreanu. Si son futur beau-frère et bras droit Ion Moţa avait été influencé dans sa jeunesse par Maurras, Codreanu le fut par la capacité d'organisation des Burschenschaften radicales allemandes pendant la crise de la Ruhr. Et c'est lui qui finit par imposer sa personnalité - déjà bien affirmée dès les premières actions antibolcheviques de 1919 - au milieu des années vingt.

Fin 1922, Codreanu se rendit en Allemagne dans le cadre d'un voyage d'études à l'université de Berlin, mais en réalité il « étudiait l'organisation étudiante allemande et autrichienne de l'activité antisé-

Hillgrüber, Hitler, König Karol und Marschall Antonescu. Die deutsch-rumänischen Beziehungen 1938-1944, Wiesbaden, Franz Steiner Verlag, 1965 (2e édition) (publié en roumain, Hitler, regele Carol şi Mareşalul Antonescu. Relatiile germano-române, 1938-1944, Bucarest, Humanitas).

4. - Traian Sandu, « Droite française, fascisme italien : influences croisées sur la Garde de Fer », dans Analele Universităţii Bucureşti, année VI, 2004, p. 61-77. 
mite $»^{5}$. Ce voyage, qui semble également prouver que Codreanu parlait allemand, puisque son père était un professeur d'allemand de lycée et que sa mère, Eliza Brauner, avait des origines bavaroises, fut d'une importance capitale pour l'organisation du mouvement antisémite en Roumanie : les souvenirs de Codreanu ainsi que les documents d'archives le prouvent. Quand il revint de l'Allemagne révolutionnaire après le début de l'occupation française de la Ruhr et de la grande inflation, les swastikas s'épanouirent sur les manteaux des étudiants roumains pour marquer les adeptes et récolter l'argent pour soutenir l'autonomie d'un mouvement ultra-nationaliste qui naquit en mars 1923, la Ligue de défense nationale-chrétienne [Liga Apărării naţională creştină, LANC], sous la direction de son vieux parrain et mentor, le professeur d'économie politique d'A.C. Cuza, un professionnel de l'antisémitisme «scientifique ${ }^{6}$ :

De même, on observe que de nombreux jeunes de la ville [sans doute Iaşi] portent comme épingles à cravate des insignes, le signe distinctif de l'association antisémite. Ces insignes auraient été apportés par l'étudiant Zelea Codreanu qui, durant son séjour à Berlin, au contact des étudiants allemands nationalistes et antisémites, a étudié leur organisation, dans le but de former ici aussi des centres étudiants, d'après le modèle de là-bas. Les insignes se vendent dans le but d'augmenter le fond de propagande antisémite, etc. .

Le récit même que Codreanu fait de son voyage en Allemagne accorde une importance particulière à ce contact. Interrogé sur la préparation d'un attentat raté contre des hommes politiques éminents et des dirigeants de la communauté juive en octobre 1923, Codreanu revient sur son sens :

Le but de mon voyage était seulement les études, mais en arrivant en Allemagne, je me suis naturellement tourné vers le problème sémitique, en achetant tous les livres qui avaient un lien avec cette question. Dès que je suis rentré, je suis allé à Iaşi, à travers Cernăuţi, où je me suis arrêté un jour et j'ai rencontré les étudiants. J'ai pris la tête du mouvement étudiant à Iaşi et ensuite j'ai essayé de présenter mon opinion à différents centres d'étudiants, à savoir : la transformation du mouve-

5. - Rapports de la Siguranţa [sécurité], 7 et 17 novembre 1922, dans Ioan Scurtu et al., Totalitarismul de dreapta în România. Origini, manifestări, evoluţie [Le totalitarisme de droite en Roumanie. Origines, manifestations, évolution], Bucarest, Institutul naţional pentru studiul totalitarismului, 1996, vol. I, 1919-1927, doc. n²4 et 25, p. 256257.

6. - Cristian Sandache, Doctrina naţional-creştină în România [La doctrine nationale chrétienne en Roumanie], Bucarest, Paideia Ştiinţe, 1997.

7. - Rapport de l'Inspectorat général de Police et de Sûreté Chişinău du 20 février 1923, ibid., doc. n³6, p. 311. 
ment étudiant universitaire en un grand mouvement national hors de l'université, parce que notre lutte intéressait toute la nation. Cette proposition a été acceptée par les centres de Iaşi et de Cernăuţi, les autres ne reconnaissant son efficacité qu'après quatre mois, au Congrès étudiant de $\operatorname{Iaşi}^{8}$.

Cette influence d'un soulèvement radical et organisé de la jeunesse allemande a certainement eu un impact sur l'avantage que Codreanu a pris sur ses camarades francophiles comme Ion Moţa, Vasile Marin ou Mihail Polihroniade, formés dans l'entourage des salons parisiens de droite du vainqueur français. Ainsi le nationalisme livresque du vieux leader de l'Action Française ${ }^{9}$ le cédait-il au dynamisme de la jeunesse frustrée et violente, adepte du coup de force révolutionnaire immédiat, adaptable à tous les pays, vaincus comme vainqueurs, frustrés et/ou déséquilibrés par les (dés)annexions à l'issue de la guerre.

Si le but de cet article n'est pas la comparaison des idéologies, lorsque les acteurs s'y livrent eux-mêmes, elle prend une dimension de considération relationnelle que l'on ne peut pas exclure. Concluons donc cette section sur l'émergence de l'idéologie légionnaire parmi les fascismes européens par un texte bref et synthétique, démontrant une inscription pleinement euro-synchrone et portant néanmoins les stigmates du retard socioéconomique et de culture politique de la Roumanie - bref, la possibilité d'un grand fascisme dans un petit pays, alors que le fascisme pose aussi la question d'une puissance internationale capable d'imposer la supériorité prétendue de sa propre nation face aux autres dans l'ultima ratio qu'est la guerre. Codreanu le reconnaît indirectement en empruntant à Mihail Polihroniade une définition qu'il présente au penseur fasciste italien Julius Evola lors d'une rencontre à Bucarest début 1938 :

D'après moi, dans le cadre du mouvement fasciste prédomine l'élément étatique, l'équivalent de la forme organisée. On ressent alors les valences formatrices de la Rome antique, indépassable en matière de droit et d'organisation politique, et dont le véritable héritier est le peuple italien. Le national-socialisme, à l'inverse, met en évidence ce qui est lié aux forces vitales : la race, l'instinct de la race, l'élément nationalethnique. Dans le mouvement légionnaire roumain, l'accent se porte sur ce qui, dans un organisme, correspond à l'élément âme, donc à l'aspect spirituel et religieux ${ }^{10}$.

8. - Interrogatoire consécutif au projet d'attentat d'octobre 1923 (ibid., doc. nº72 du 31 octobre 1923).

9. - Traian Sandu, « De Charles Maurras à Lucien Rebatet : un alibi de droite français pour le fascisme roumain de la Garde de fer ? », dans Olivier Dard et Michel Grunewald (dir.), Charles Maurras et l'étranger - l'étranger et Charles Maurras. L'Action Française - culture, politique, société II, Berne, Peter Lang, 2009, p. 169-191.

10. - Julius Evola, Naţionalism şi asceza [Nationalisme et ascèse], Bucarest, 
Or Codreanu revendique dans Pentru legionari/La Garde de fer le fond commun du fascisme générique, mais l'ajuste selon la variable socioculturelle d'une logique inversement proportionnelle. Ainsi la variante la plus primitive, la race, correspond-elle au principe d'intégration fasciste du pays le plus développé, l'Allemagne, dont la société civile est la plus autonome par rapport à un État décentralisé récent et a créé des mythes à base de racisme biologique portés par un tissu associatif prolifique au tournant du siècle, en attendant qu'ils soient institutionnalisés par l'État-mouvement nazi. Moins développée, l'Italie fournit l'image de masses moins cultivées politiquement, donc moins totalement mobilisées par un fascisme ayant besoin de l'institutionnalisation étatique afin de faire face au dynamisme des chefs locaux - les ras, selon la dénomination héritée de la guerre d'Éthiopie - au sein du mouvement, d'où l'insistance mussolinienne sur l'État. Enfin, le Mouvement légionnaire naît parmi la population estudiantine moderne et urbaine d'un pays européen arriéré, périphérique, mais en voie de développement, dont la communication politique en vue de la massification doit encore passer par les vecteurs traditionnels de la religion et du sentiment monarchique pour atteindre une population majoritairement rurale encore soumise aux pouvoirs traditionnels de l'Église et de l'État royal. De même, en termes de réalisation majeure concrète, si tous les fascismes ont profondément modifié l'esprit et la culture politique de leur espace national, seuls les fascismes-régimes majeurs ont réussi à marquer le destin de l'Europe dans une gradation sanglante décroissante de l'ingénierie sociale totalitaire et éliminationiste des « inadaptés ». Le génocide juif procède du fascisme antisémite hypermoderne de la grande puissance allemande, l'homogénéisation violente, standardisée et enthousiaste d'un pays très hétérogène en matière de développement par le principal opérateur disponible, l'État italien dynamisé par le mouvement fasciste, et la Roumanie en voie de développement fournissant une synthèse fasciste entre l'État moderne agressif et la religiosité d'une société traditionnelle, mais qui finalement échoue à dépasser le quart de l'électorat et à arracher le pouvoir.

\section{Après la « Machtergreifung », un intérêt mutuel mais circonspect}

Avec la création du mouvement de la Légion de l'Archange Michel en 1927 et de sa façade politique de la Garde de fer en 1930, Codreanu

Fronde, 1998, p. 42-43. Voir la reprise de cet argument dans la défense de Codreanu du 23 mai 1938 à son procès pour trahison et rébellion, Scurtu et al., op. cit., vol. V (1938-1940), doc. n³8, p. 98-112, ici p. 112. 
passa son temps à définir son idéologie. Les relations avec l'Allemagne et les Allemands de Roumanie furent plutôt discrètes durant cette période.

En 1933, Codreanu fit preuve d'une attitude amicale, organisant un contre-boycott des magasins juifs pour répondre au boycott juif des produits allemands - consécutif au soutien apporté par certains Deutschnationale à la Machtergreifung ${ }^{11}$ lors d'une réunion à Rădăuţi, en Moldavie, à laquelle assistaient également les Nazional Sozialisten Deutsch Rumänen [sic !] ${ }^{12}$. Cette initiative avait probablement aussi pour but de contrebalancer les manifestations pro-hitlériennes bien plus bruyantes de la part d'A.C. Cuza, de son fils Gheorghe, qui avait été reçu par Hitler, l'aile jeune de la LANC formant des bataillons d'assaut, les « lanciers ", sur le modèle allemand, pour molester la population juive et livrer des combats contre le concurrent légionnaire. D'autres chefs de groupuscules radicaux comme Ştefan Tătărescu, chef du Parti national socialiste roumain - à ne pas confondre avec son frère aîné, Gheorghe Tătărescu, le futur Premier ministre ! -, rencontrèrent Hitler durant leur séjour berlinois en août-septembre 1933 et en rapportèrent une impression puissante, mais aussi une servilité13 que l'on chercherait en vain chez Codreanu. Cela ne l'empêchait pas d'effectuer des réunions communes avec les organisations nazies de Transylvanie et de promettre une coopération économique harmonieuse, en s'élevant contre le boycott des produits allemands ${ }^{14}$. Par conséquent, la répression envisagée par le Premier ministre libéral Ion Gheorghe Duca contre la Garde de fer semblait injustifiée pour ce motif précis.

Si Codreanu n'entretenait certainement pas les rapports de soumission envers l'Allemagne des petits groupuscules roumains, il avait néanmoins compris l'importance nouvelle du facteur allemand. La diplomatie roumaine préparait son intégration à l'axe Paris-Moscou en gestation depuis l'arrivée au pouvoir de Hitler ${ }^{15}$, et les partis démocratiques

11. - Article sans titre dans Axa, 9 avril 1933, dans Scurtu et al., Ideologie şi formaţiuni de dreapta în România [Idéologie et formations de droite en Roumanie], Bucarest, Institutul naţional pentru studiul totalitarismului, vol. III, 5 janvier 1931-7 juin 1934, 2002, ici doc. n66, p. 169-170.

12. - Rapport du procureur général de la Cour d'appel de Cernăuţi au ministre de la Justice du 24 avril 1933, ibid., doc. n68, p. 171-172.

13. - « Colonel Tătărescu în Germania » [Le colonel Tătărescu en Allemagne], dans Deutsche Zeitung Bessarabiens du 30 septembre 1933, ibid., doc. n90, p. 211212 : «Votre chef... est appelé à sauver non seulement l'Allemagne, mais le monde entier $\gg($ p. 211).

14. - Note n8162 de la Direction de la police et de la sûreté du 24 avril 1933, ANIC, Interne-Diverse, vol. 8/1933, 2e partie, f. 39-42.

15. - Voir, entre autres, notre article : «La Roumanie sur l'axe Paris-Moscou, 19331937 : sécurité européenne et coopération militaire », dans Anne-Claire Bonneville-de Gayffier, Sécurité et coopération militaire en Europe, 1919-1955, Paris, L'Harmattan, Cahiers d'Histoire de Saint Cyr-Coëtquidan nº1, 2004, p. 249-267. 
donnaient des gages à Paris dans ce sens : une des dimensions de ces garanties pouvait être la répression, sur la scène politique intérieure, des mouvements de la droite radicale pro-allemande ou, du moins, antisoviétique. Mais les tensions qui traversaient les relations internationales pouvaient aussi ouvrir des possibilités au Mouvement légionnaire, car une bonne partie des droites était hostile, sinon à une normalisation des relations, du moins à une alliance avec l'Union Soviétique comportant des clauses de passage de l'Armée rouge par le territoire roumain. À mesure que cette politique menée par le ministre des Affaires étrangères Nicolae Titulescu se précisa, les positions hostiles à l'adhésion à l'axe Paris-Moscou de Codreanu trouvaient une compréhension croissante au sein de la droite traditionnelle.

Toutefois, cette convergence entre légionnaires et droite traditionnelle en politique internationale ne signifiait pas automatiquement, loin s'en faut, un rapprochement domestique. Une compétition pour obtenir le cachet de la protection allemande pouvait émerger entre groupes de la droite radicale, sans même parler de la volonté du roi et des partis de gouvernement de ne pas voir la diplomatie officielle d'un rééquilibrage des relations avec la France et l'Allemagne parasitée par les fidélités intérieures des mouvements radicaux envers Berlin. La carte allemande pouvait donc s'avérer à double tranchant, voire dangereuse en cas d'impression que la Légion remportait non seulement un succès populaire intérieur, mais aussi une éventuelle faveur du nouvel arbitre européen.

Codreanu donna une double réponse d'indépendance et de sympathie envers l'Allemagne hitlérienne. L'indépendance était traitée là où le bât blessait le plus, le révisionnisme territorial et le financement allemands :

Supposons que Hitler veuille nous faire la guerre, et nous prendre la Transylvanie. Nous, Roumains, pour défendre la Transylvanie contre les Allemands, il faut d'abord que nous soyons forts. Et pour être forts, il faut que nous nous délivrions des juifs ; que nous résolvions, nous aussi, le problème juif, que nous rendions courage à notre peuple épuisé par les juifs, et mis dans l'impossibilité de se défendre. Avec la juiverie qui nous empoisonne le cœur et nous suce le sang, nous ne pouvons avoir ni armes, ni âme, ni force.

[...] Enfin, nous recevons de l'argent, nous sommes «stipendiés », nous sommes «à la solde » de l'Allemagne. Nous répondons : «A. C. Couza [sic : Cuza] attaque les juifs depuis 1890, nous depuis 1919, 1920, 1921, 1922, alors qu'il n'était pas encore question de Hitler. »

Ces juifs ne sont que des serpents venimeux ${ }^{16}$.

16. - Corneliu Zelea Codreanu, La Garde de Fer, Paris, Ed. Prométhée, 1972, p. 418. 
Même si cette virulente affirmation d'indépendance trouvait un fondement dans le caractère autochtone du fascisme roumain, sa violence même signifiait aussi un début d'intérêt mutuel entre les mouvements nationalistes radicaux d'Europe. L'Allemagne pouvait d'autant moins ignorer la scène politique roumaine que la minorité allemande y possédait une représentation que les nazis tentaient de noyauter et que la politique d'autarcie de Hitler visait, entre autres, la reconstitution d'une Mitteleuropa pro-allemande, avec une mention spéciale pour les céréales et surtout pour le pétrole roumains. Il n'est pas impossible que, très tôt, Hitler ait souhaité un regroupement de certains partis de droite pour détenir un levier de pression sur le pouvoir roumain. De ce point de vue non plus, Codreanu ne pouvait lui donner aucune satisfaction, ainsi qu'il ressort d'une note de police de cette période :

Au sujet d'une fusion de la «Garde de Fer » avec l'un des partis ou groupements politiques, il ne peut pas en être question. Je ne peux même pas admettre de discussions sur ce thème, car je ne peux pas admettre que le Commandement politique unique de la «Garde de Fer» soit modifié dans le sens qu'une deuxième personne en-dehors de moi ait aussi un droit en ce qui concerne les décisions ou les directives données aux organisations subordonnées ${ }^{17}$.

À l'intérieur, l'interdiction de la Garde de fer en décembre 1933, l'assassinat immédiat du Premier ministre Ion Gheorghe Duca et le procès du printemps 1934 relancèrent en fait l'activité ; à l'étranger, l'assassinat fut amplement critiqué, un débat indirect étant suscité entre la France et l'Allemagne. Quelle plus belle preuve de l'autonomie politique d'un fascisme roumain autochtone que les accusations de l'Action Française envers l'Allemagne nazie d'avoir commandité l'assassinat et la défense de la presse allemande outrée ${ }^{18}$ ? Une semaine après l'attentat du 29 décembre, l'historien membre de l'Action Française Jacques Bainville ne cacha pas son inquiétude devant l'activisme hitlérien en Europe centrale :

Jean Duca n'a pas été frappé seulement parce qu'il avait dissous la ligue des Gardes de Fer, mais parce qu'il était fermement attaché à la Petite Entente et à la France. Ou, ce qui revient au même, il frappait cette association hitlérienne en raison de la ligne qu'il suivait, pour la politique extérieure de la Roumanie, avec M. Titulesco ${ }^{19}$.

17. - Note d'information de la Direction de la Police de sûreté du 20 octobre 1933, Scurtu et al., op. cit., doc. n' 96 , p. 219-220.

18. - Voir notre article «Droite française, fascisme italien : influences croisées sur la Garde de fer », Analele Universităţii Bucureşti, année VI, 2004, p. 61-77.

19. - Jacques Bainville, «Après le meurtre de Sinaia », dans La Russie et la barrière de l'est, Paris, Plon, Ed. d'histoire et d'art, p. 228-229. 
Or, alors qu'« aucun des journaux de l'Italie fasciste ne trouva un mot pour condamner l'assassinat de l'ex-Premier ministre Duca $»^{20}$, la presse allemande réprouva l'assassinat et s'indigna des accusations de collusion, à juste titre d'ailleurs :

Dans de longs télégrammes de Budapest et de Bucarest, la presse allemande a minutieusement relaté les circonstances dans lesquelles a été commis l'assassinat contre le ministre I.G. Duca, en montrant qu'il est l'œuvre de l'organisation anarchique de la Garde de fer. [...] Unanimement, les journaux allemands ont condamné l'assassinat et ont mis en évidence l'ensemble des mesures prises par le gouvernement roumain pour assurer l'ordre et la tranquillité dans le pays. [...] Berliner Börsen Zeitung (31.XII.933) Après avoir montré que par l'assassinat d'I.G. Duca l'autorité de l'État roumain n'est pas mise en péril, il repousse certains commentaires faits par la presse française qui a attribué l'attentat à l'influence directe du national-socialisme allemand et souligne que les bonnes relations entre l'Allemagne et la Roumanie ne peuvent pas être atteintes par de telles tentatives condamnables ${ }^{21}$.

\section{Refus légionnaire d'une convergence des droites roumaines ad usum Germaniae...}

Les années 1934-1935 enregistrèrent les premiers tâtonnements dans un rapprochement avec l'Allemagne. Codreanu accorda une interview au Berliner Illustrierte Nachtausgabe de mai 1934, dans laquelle « le futur homme de la Roumanie » transmettait « à Hitler et au peuple allemand [son] salut ${ }^{22}$. L'indépendance du mouvement fasciste roumain par rapport aux deux grands modèles et son attachement à l'antisémitisme allemand se lisent dans le choix que fit Moţa lors du congrès des Comités pour l'universalité de Rome (CAUR), sorte de vitrine internationale du régime fasciste dont les Allemands étaient exclus, à Montreux en décembre 1934. A priori, le révisionnisme territorial d'aucun des deux régimes ne devait lui convenir, mais la politique italienne pouvait lui répugner à double titre comme pro-hongroise et pas assez antisémite. La question diplomatique étant trop délicate à aborder et risquant de soulever des problèmes avec la délégation hongroise, ce fut sur le terrain fertile de l'antisémitisme que Moţa livra bataille. La motion sur la question juive qu'il présenta à Montreux se solda par une déclaration ambivalente : dans la ligne italienne, « le Congrès déclar[a] que la question juive ne pou[v]ait pas se traduire par une campagne universelle de haine contre les juifs »; mais les antisémites obtinrent la recon-

20. - Revue de presse, ANIC, CR, vol. 119/1934, f. 3.

21. - Ibid., f. 18.

22. - Dossier de presse du 14 mai 1934, ANIC, CR, vol. 72/1934, f. 8. 
naissance du problème juif comme celle d'« un État dans l'État » et d'une minorité révolutionnaire internationaliste, entraînant la décision de la combattre ${ }^{23}$. Moţa évoqua l'affaire avec un correspondant de l'organisation de propagande antisémite internationale du Welt-Dienst ${ }^{24}$, dont le directeur, Ulrich Fleischhauer ${ }^{25}$, avait établi le siège à Erfurt. Les Allemands, en délicatesse avec les Italiens au moment de la crise autrichienne de juillet 1934, n'avaient pas été invités à Montreux, où Moţa dut représenter la tendance antisémite du fascisme européen et se vanta d'avoir arraché la motion aux Italiens ${ }^{26}$. À ce propos, il distingua clairement l'approche germano-roumaine raciste de l'approche francoitalienne, "spiritualiste », mais il conseilla à son correspondant de modérer la dimension uniquement raciste de son antisémitisme afin de le rendre plus acceptable aux yeux des Italiens et de permettre aux Allemands de participer aux futurs congrès de la CAUR ${ }^{27}$.

Après ce service rendu, le même Moţa dut justifier auprès de ses amis allemands le refus de la Légion d'obtempérer aux injonctions de Hitler de rapprochement entre partis et mouvements de droite. Il émit de sérieux doutes quant à la capacité de Vaida-Voevod à insérer l'antisémitisme dans un projet global de révolution fasciste et de combativité radicale contre le régime qui protégeait les juifs ${ }^{28}$. Derrière Vaida-Voevod se profilait le problème de la collaboration avec le concurrent insupportable et pourtant hautement germanophile, A.C. Cuza. Nous avons vu les démarches des représentants de la communauté allemande de Roumanie, ainsi que des nazis roumains avec Ştefan Tătărescu ; VaidaVoevod et Octavian Goga étaient les autres personnalités qui pouvaient participer à la restructuration de la droite nationaliste selon des perspectives qui aboutissaient à l'autorité du roi ou aux souhaits de Berlin. Mais il s'agissait de fascismes labiaux, dépourvus de la mobilisation des mythes et des masses indispensables au fascisme véritable, qui refusèrent finalement une telle manipulation.

23. - Note de la Direction générale de la Police du 21 janvier 1935, Scurtu et al., op. cit., doc. $\mathrm{n}^{\circ} 24$, p. 81.

24. - Ion Motza, Corrispondenza col Welt-Dienst [1934-1936], Parme, All'insegna del Veltro, 1996 ; traduction roumaine : Corespondenţa cu Welt-Dienst, Munich, Ed. « Ion Mării », 2000, p. 74-78, également cité dans Scurtu et al., op. cit., doc. $\mathrm{n}^{\circ} 28$, p. $84-86$.

25. - Voir la page que lui consacre Richard Alan Nelson sur Wikipedia (consulté le 22 avril 2010) : http://en.wikipedia.org/wiki/Ulrich_Fleischhauer

26. - Ion Moţa, Corespondenţa cu Welt-Dienst..., loc. cit.

27. - Ibid. : "Même une entente italo-allemande sur ce problème serait plus facile à faire si l'on ne présentait pas la question juive comme explicable seulement par la doctrine raciste et donc de prétendre la liaison indissoluble des actions anti-judaïque et raciste ».

28. - Moţa, « Numerus clausus », Cuvântul studenţesc du 20 mars 1935, Scurtu et al., op. cit., doc. n³3, p. 88-91. 
La diplomatie allemande souhaitait un rassemblement des forces de droite afin de peser sur la politique du roi et de faire éclater à un moment délicat le lien franco-roumain ${ }^{29}$. La tenue de la réunion de la Petite Entente début mai 1935 à Bucarest devait donner son feu vert à la signature du pacte soviéto-tchécoslovaque du 16 mai, immédiatement après le pacte franco-soviétique du 2 mai ; l'étau diplomatico-militaire des deux puissances, censé converger vers Berlin par la Tchécoslovaquie, avait besoin du droit de passage des troupes soviétiques auquel la Pologne se refusait et qui ne pouvait plus se faire que par la Roumanie, avec l'angoisse d'une occupation soviétique et d'une soviétisation. Les légionnaires, censés être proches de Berlin, étaient rendus responsables des difficultés rencontrées par la signature d'un pacte soviéto-roumain, alors qu'en réalité l'ensemble de l'opinion de droite se méfiait de cette politique menée par un Titulescu de plus en plus isolé, à commencer par le $\mathrm{roi}^{30}$. Une note rapportant les propos d'un responsable légionnaire rappela l'indépendance d'esprit de Codreanu face à Berlin et la relation équilibrée que la Légion désirait que la Roumanie établît avec les États fascistes :

Un autre axiste [membre du «nid »- cellule politique légionnaire - intellectuel $A x a$ ], qui s'occupe des problèmes ayant trait à la politique extérieure, montre que Corneliu Zelea Codreanu est dans le juste ton des exigences nationales concernant la politique extérieure, car il n'est pas un partisan fidèle de l'hitlérisme, comme le sont les cuzistes, ni du fascisme vers lequel penchent M. Goga et d'autres nationalistes. Le chef de la Garde, et avec lui les autres dirigeants, sont pour une politique extérieure dictée seulement par les intérêts supérieurs du pays, et non par les intérêts mesquins de groupe. Ils sont pour un rapprochement avec l'Italie et l'Allemagne, mais à condition que ce rapprochement soit utile au pays et se produise seulement après que ces pays ont fourni la preuve qu'entre la Roumanie et ses ennemis, ils choisiraient l'amitié avec la Roumanie, tant dans les questions économiques que dans les questions purement politiques ${ }^{31}$.

29. - Ioan Chiper, România şi Germania nazistă : Relaţiile româno-germane intre comandamente politice şi interese economice: (ianuarie 1933 - martie 1938), Bucarest, Ed. Elion, 2000, p. 149.

30. - Voir une référence certes plus tardive du journal de Carol II, Între datorie si pasiune, op. cit., p. 160-161, le 30 mars 1937 : « Pour nous, l'URSS reste encore le point faible. Tous, à commencer par moi, et en exceptant quelques nationaux-paysans et Tituoxi [Titulescu], nous sommes fermement contre des rapports trop proches avec elle. Mais dans cette position, nous avons, au moins, un peu de tranquillité de leur part, sinon leur amitié. Si, ainsi que le désirent certains, nous nous rapprochons de l'Allemagne et de l'Italie, la situation à l'égard de l'URSS devient précaire et nos autres alliances aussi ».

31. - Note du 24 septembre 1935, CNSAS, vol. D8906, f. 103-104, ici f. 104. 
Cela n'empêcha pas certains légionnaires d'adopter des discours violemment révolutionnaires, modernistes et d'un racisme fondé sur le sang et le déterminisme biologique comme chez Alexandru Cantacuzino, s'accompagnant de phrases admiratives à l'égard des « races aux âmes impériales, dominatrices. Ce sont l'Italie et l'Allemagne $»^{32}$. Évidemment, l'Italie mussolinienne, qui venait de signer en janvier un traité avec la France, était certainement moins suspecte que l'Allemagne, mais elle représentait un autre système d'alliances antiallemand alternatif, et en fait concurrent, de l'alliance de revers du couple franco-soviétique. Donc même la référence italienne n'était pas si rassurante pour la France lorsqu'elle était exprimée par un fasciste roumain, puisque la Roumanie avait, dans le plan d'ensemble français, un rôle dans l'alliance franco-soviétique, et non dans l'alliance francoitalienne. Mais un légionnaire pouvait-il s'attarder à ce genre de menues considérations, lorsqu'il exprimait le fantasme d'« élever ce peuple au rang de grande puissance en Europe $»^{33}$ ?

Ce n'était pas ce qu'attendait Berlin, ni sur le plan international ni en politique intérieure, où Moţa refusa à nouveau à ses correspondants du Welt-Dienst une intégration de la Légion et des autres droites pour faciliter l'action de l'Allemagne en Roumanie. Les raisons sont explicites :

Tous les autres mouvements, Cuza, Goga, Vaida, n'ont aucune valeur : ils peuvent, dans le meilleur des cas, constituer une introduction à la victoire légionnaire, la seule qui résoudra le problème juif de façon radicale. [...] J'ai déjà été clair, je crois, sur la raison pour laquelle je suis contre une fusion des organisations, car celle-ci altère l'unité, lorsqu'elle ne se fait pas entre hommes avec le même contenu spirituel, dans le sens de la lutte hérö̈que. Notre force réside dans notre unité et notre esprit de sacrifice, dans la croyance totale en notre chef. Une fusion détruirait tout cela, croyez-moi, même si une lettre ne me permet pas de vous éclairer plus en détail. Nous restons amis seulement avec ceux qui sont de bonne foi, mais sans faire une union totale avec eux, justement pour éviter les futurs compromis, trahisons et faiblesses ${ }^{34}$.

32. - Alexandru Cantacuzino, « Românul de mâine » [Le Roumain de demain], conférence tenue au congrès général estudiantin d'avril 1935 de Craiova, Scurtu et al., op. cit., doc. $\mathrm{n}^{\circ} 39$, p. 99-105, ici p. 100.

33. - Ibid., p. 105.

34. - Lettre de Moţa du 15 juin 1935 à Farmer de la direction du Welt-Dienst, ibid., doc. n45, p. 111-113, ici p. 112. 


\section{... Mais soutien gardiste à l'Axe fasciste sur le plan international}

L'intrusion de la Légion dans le débat sur la politique étrangère roumaine n'était pas, nous l'avons vu, une surprise, tant la situation de petite puissance sous tutelle et les énormes pressions géopolitiques plaçaient la question au centre d'un débat public idéologisé. L'option claire en faveur de l'Axe en voie de constitution se fit jour à travers la circulaire du 30 mai 1936 sur la politique extérieure ${ }^{35}$. Codreanu y exprima son inquiétude quant à la diplomatie roumaine tenue entre « les mains, très débiles, [de] M. Titulescu »; le rapprochement de la Russie recevait cette fois une réprobation idéologique rédhibitoire, sans égards pour la politique antirévisionniste de l'axe Paris-Moscou et pour le jeu des puissances :

Le rapprochement avec la Russie.

I. C'est un geste de trahison que le peuple roumain fait à l'égard de Dieu et à l'égard de l'ordre moral du monde et à l'égard des peuples qui sont au service de cet ordre, dans la guerre avec les puissances destructrices du mal. Honneur à ces peuples ${ }^{36}$.

Évidemment, la réflexion géopolitique n'était pas absente de son raisonnement et était, d'ailleurs, assez largement partagée par l'ensemble de la droite roumaine. Que la Roumanie fût prise entre le risque de bolchevisation en cas de passage des troupes soviétiques et le risque de démembrement de la part des puissances fascistes en cas de victoire sur le couple franco-russe était un classique de la réflexion stratégique ${ }^{37}$. La motivation idéologique radicale au détriment de la pensée stratégique nationale et d'une réflexion plus équilibrée entre risque de domination soviétique et risque de domination de l'Allemagne nazie était toutefois propre à Codreanu. Le choix clairement favorable aux puissances de l'Axe en voie de constitution l'était aussi, alors que le roi privilégiait un rééquilibrage prudent ${ }^{38}$, en quittant avec précaution seulement l'axe Paris-Moscou que l'agrarien de gauche Ion Mihalache préconisait toujours dans son adresse solennelle après le congrès étudiant de TârguMureş, qui avait vu la radicalisation à droite et la quasi-rupture avec le

35. - « Circulaire-politique extérieure », Codreanu, Circulări şi manifeste..., op. cit., p. 74-75.

36. - Ibid.

37. - Voir, par exemple, la lettre personnelle du 14 juillet 1936 d'Octavian Goga à Carol II, ANIC, CR, vol. 7/1936, f. 3-9.

38. - Voir notre article déjà cité « La Roumanie sur l'axe Paris-Moscou, 19331937 : sécurité européenne et coopération militaire », dans Anne-Claire Bonneville-de Gayffier (dir.), Sécurité et coopération militaire..., op. cit. 
monarque, encore complaisant jusque-là ${ }^{39}$. À Paris, son alter ego, l'économiste Virgil Madgearu, fit des déclarations similaires, attribuant les succès de la Légion à la main de Berlin et situant le Parti national paysan dans la double hostilité au fascisme et au communisme ${ }^{40}$. Le désir de dédouaner le peuple roumain en général de la dynamique fasciste relève du souci des partis modérés de préserver la situation internationale de la Roumanie à l'égard des puissances occidentales, exactement comme les partis d'extrême droite désiraient revaloriser l'image de la Roumanie auprès des deux puissances fascistes. La radicalisation du débat intérieur roumain se structurait de plus en plus selon des clivages internationaux, non pas tant parce que Berlin ou Moscou auraient soutenu ou, moins encore, stipendié respectivement la Garde de fer ou le Parti national paysan, mais parce que, précisément, chacun des deux partis avait reconnu en l'autre le vecteur d'une dynamique puissamment ancrée dans un messianisme idéologique séculier, populiste de droite pour l'un, démocratique pour l'autre.

Si le torchon brûlait depuis longtemps entre Carol II d'un côté et le ministre des Affaires étrangères de l'autre ${ }^{41}$, les légionnaires furent tôt au courant des conditions de la chute à venir de Titulescu : lors d'une rencontre entre chefs légionnaires du 13 juillet, le général Gheorghe Cantacuzino affirma que «"le roi n'[était] plus d'accord avec lui [Titulescu]" ; voyant les erreurs qu'il a[vait] faites dans la politique étrangère du pays, il attend[ait] le moment indiqué pour l'écarter de la tête du ministère des Affaires étrangères ${ }^{42}$.

Mais cette évolution n'eut aucun impact sur la sévérité des critiques légionnaires. Codreanu reprit la question dans des termes proches de ceux qui avaient précédé la destitution de Titulescu fin août avec sa

39. - Mémoire cité de Mihalache au roi de mai 1936, Scurtu et al., op. cit., doc. n93, ici p. 191.

40. - « Mişcările extremiste nu au rădăcini în România. Declaraţiile făcute de D. Virgil Madgearu ziarului Le Petit parisien » [Les mouvements extrémistes n'ont pas de racines en Roumanie. Déclarations effectuées par M. Virgil Madgearu au journal Le Petit parisien], déclarations reprises par Dreptatea du 1er juillet 1936, ibid., doc. n96, p. 194-195.

41. - Voir l'échange de télégrammes par lesquels Tătărescu proteste contre l'affirmation du ministre des Affaires étrangères français Yvon Delbos selon lequel, impressionné sans doute par les déclarations de Madgearu à Paris, la situation aurait été sur « le seuil de la complète hitlérisation de la Roumanie » (tél. chiffré n³6020 du 30 juin 1936 de Tătărescu à Titulescu, ANIC, CR, vol. 95/1936, f. 1-3). Titulescu fit preuve d'une grande irritation dans sa réponse, car le Premier ministre et le roi le dessaisissaient ainsi de ses prérogatives diplomatiques et s'insurgeaient contre l'allié français (tél. $\mathrm{n}^{\circ} 151 \mathrm{du}$ 1er juillet 1936, ibid., f. 4-6).

42. - «Un conseil des leaders légionnaires », note n²081 du 14 juillet 1936 de la DGP, ANIC, DGP, vol. 46/1936, f. 221. 
lettre ouverte au roi du 5 novembre $1936^{43}$. Le ton solennel et urgent, l'adresse au roi, aux hommes politiques et à l'opinion entière correspondent à la remilitarisation de la Rhénanie en mars 1936 et à la structuration de l'Axe Rome-Berlin lors de la guerre d'Espagne. Plus précisément, Codreanu réagissait à une réplique violente de Mussolini contre le soutien apporté par la Roumanie aux sanctions prises contre l'Italie à la suite de la guerre d'Éthiopie. Le premier point demandait que les responsables, y compris le roi, répondissent «sur leur tête » de leur politique étrangère. Dans le deuxième point, il menaça d'insoumission en cas de guerre aux côtés de l'axe Paris-Moscou, et même de révolte contre le personnel qui les y aurait amené, le tout au nom de raisons idéologiques - ne pas « [lutter] sous le signe de Satan » bolchevique - qu'il développa dans le troisième point en opposant « les États des révolutions nationales, qui luttent pour la défense de la Croix et d'une civilisation millénaire et le bolchevisme et ses annexes [...] [qui] seront anéantis par les armées de la Croix et de l'ordre naturel du monde. Si les politiciens roumains nous mènent dans leur camp, la Roumanie sera effacée de la carte de l'Europe. » Le point quatre justifiait la dureté du discours de Mussolini à l'égard de la Roumanie après le soutien apporté aux sanctions. Le point cinq déplaçait le débat sur le plan intérieur, comparant l'acharnement contre l'Italie à celui exercé contre la jeunesse nationaliste. Enfin, dans le sixième et dernier point, il appelait à nouveau à la révolte nationale contre l'ennemi intérieur, grâce à un front de « tous ceux qui se trouvent aujourd'hui sur la ligne du destin et de l'Histoire nationale ».

Si l'intention de Codreanu tendait explicitement au rassemblement des forces nationalistes, le ton adopté et les moyens dont il menaçait un roi qui se trouvait aussi sur des positions de rééquilibrage diplomatique témoignent plutôt de son désir de profiter des changements de puissance sur la carte diplomatique pour mettre le souverain en difficulté. Sans appeler à l'appui des forces étrangères, Codreanu entendait au moins signifier aux puissances de l'Axe qui était leur véritable supporter idéologique sur la scène politique roumaine et leur éventuel interlocuteur le plus adapté. La politique étrangère devenait ainsi un levier puissant dont le chef fasciste entendait jouer pour démultiplier sa capacité sur la scène intérieure. Codreanu travaillait d'ailleurs à une note définissant les rapports entre la Légion et la monarchie d'où il ressortait que « le rôle rempli par le roi, en tant que monarque constitutionnel, [...] ne [devait] pas se mêler de la vie privée [sic] et politique du pays ${ }^{44}$.

43. - « Mémoire adressé à S.M. le Roi, aux hommes politiques et au pays », Codreanu, Circulări şi manifeste..., op. cit., p. 97-101.

44. - Note n 14726 du 5 décembre 1937, ANIC, M.I.-Diverse, vol. 5/1937, f. 150151, ici f. 150. 
Codreanu prit donc une initiative articulant les deux plans, intérieur et extérieur : il décida le départ de sept cadres légionnaires de haut niveau pour le front espagnol afin de combattre avec les nationalistes et leurs alliés allemands et italiens pendant un mois ${ }^{45}$. Au mois de janvier, Ion Moţa et Vasile Marin y perdirent la vie, ce qui donna l'occasion à Codreanu d'une énorme opération de propagande lors de leur enterrement en février, à laquelle les Allemands furent associés lors du passage du convoi mortuaire par Berlin, où les corps reçurent les honneurs de la part des Nationaux-Socialistes.

Un début de réaction publique vint avec la réponse de Tătărescu à l'interpellation lue par le jeune agrarien antifasciste Armand Călinescu au sujet de la participation des ministres étrangers à l'enterrement : ce fut une tentative de sauver la dignité nationale face à cette intrusion des puissances de l'Axe dans la politique intérieure au bénéfice de la Légion, tout en ménageant ces puissances dont la diplomatie roumaine devait avoir un besoin croissant, mais sans en laisser la gestion des relations aux légionnaires 46 .

En effet, une dépêche de Wilhelm Fabricius, le ministre d'Allemagne à Bucarest depuis 1936 - inscrit au NSDAP seulement en 1937, par opportunisme donc-, prouva qu'il avait - néanmoins - bien compris ce qui distinguait la Garde de fer du reste de l'extrême droite dont les émissaires se succédaient à Berlin pour demander des subventions et un soutien moral : «Les seuls qui méritent de l'appui et de l'encouragement sont [...] les légionnaires de la Garde de fer qui, justement à cause de leur âpreté de caractère et d'idées, sont soumis à la persécution, enfermés et poursuivis sans pitié $\gg^{47}$. Les autorités allemandes avaient appris à apprécier l'esprit de sacrifice des légionnaires lors du rapatriement des corps de ceux tombés en Espagne. En mars, le gouvernement et le roi firent comprendre à Berlin qu'un appui apporté aux légionnaires pouvait avoir de graves conséquences sur les relations germano-roumaines ${ }^{48}$.

45. - Lettre de Moţa à sa famille du 1er décembre 1936, Testamentul lui Ion Moţa, Munich, éd. « Ion Mării », coll. Europa, p. 21-24.

46. - Monitorul oficial, IIIe partie, n²8 du 3 mars 1937, p. 1036-1037, cité dans Scurtu et al., op. cit., doc. ${ }^{\circ} 159$, p. 270-272 : « [C]'est une défaite de tous les principes et de tous les usages internationaux (applaudissements vifs et prolongés sur les bancs de la majorité et sur les bancs du Parti national paysan). Le gouvernement prendra sérieusement en considération la situation créée. Je me dépêcherai d'ajouter que cette situation, créée par des actions à caractère personnel, ne peut avoir aucune répercussion sur les rapports entre notre pays et les pays représentés que nous souhaitons de plus en plus étroits et de plus en plus amicaux. (Applaudissements vifs et prolongés sur les bancs de la majorité). »

47. - « Informations au sujet d'un nouveau rapport de Fabricius », note n³0 (621) du 8 mai 1937 de la DGP, ANIC, DGP, vol. 17/1937, f. 20-21, ici f. 21.

48. - Chiper, România şi Germania nazistă, op. cit., p. 222. 
Cela n'empêcha pas les représentants de l'Allemagne à Bucarest de faire montre d'une sympathie croissante à l'égard des légionnaires. Une autre note provenant du bulletin d'informations de la légation d'Allemagne et établie par l'attaché de presse Hermann von Rittgen, qui devait jouer également un rôle de renseignement, fournissait des estimations pour les élections qui s'avèrent, rétrospectivement, d'une stupéfiante pertinence : si les résultats du Parti national libéral au pouvoir n'ont aucune importance en raison, comme il est dit, des trucages, les $15-16 \%$ des Nationaux paysans, les $15-17 \%$ de Tout pour le pays, les $10-11 \%$ des Nationaux chrétiens sont parfaitement évalués ${ }^{49}$. Toutefois, là où la note pécha sérieusement dans l'évaluation du fascisme roumain, c'est lorsqu'elle attribua aux opportunistes de droite et à Carol II l'incapacité d'une union des forces de droite ${ }^{50}$, et non à la Légion, véritable facteur de dissolution de la droite traditionnelle. Dans une autre note, il donna une définition enthousiaste de la Légion comme soulèvement populiste, reprenant les « mots mêmes de Corneliu Codreanu, qui lui [avaient] été transmis par écrit, à l'occasion d'une enquête récente provoquée par les procès anti-légionnaires ${ }^{51}$. Tous ces documents en provenance de Bucarest ne signifient pas que Berlin adoptait la même approche à l'égard de la Légion.

\section{L'assassinat de Codreanu, une désagréable surprise pour Hitler}

Le succès des légionnaires aux élections de décembre 1937 - troisième parti avec plus de $15,5 \%$ des voix - plongea la Roumanie dans le trouble politique et la violence de rue. Le pouvoir en profita pour se débarrasser de Codreanu lors d'un procès politique largement truqué durant lequel l'accusation d'intelligence avec une puissance étrangère - l'Allemagne, tacitement - joua un rôle notable. Le juge militaire fit jouer l'article portant sur la trahison en établissant une liste des circulaires de Codreanu qui témoignaient de sa connaissance de documents policiers secrets concernant la sûreté de l'État ${ }^{52}$. Sa conclusion fut que

49. - «La situation politique intérieure de Roumanie vue par la légation allemande. D'après le bulletin d'informations établi par Hermann von Rittgen et Ioseph [sic] Ortmann au 12 mai 1937 », note n 30 (2931) du 17 mai 1937 de la DGP, ANIC, DGP, vol. 17/1937, f. 30-33.

50. - Ibid. : «Les derniers essais de fusion de Messieurs Vaida, Goga, Manoilescu ne sont pas sincères » (f. 32) ; «Sa faute [celle du roi Carol] est seulement qu'il n'insiste pas plus pour la fusion des nationalistes, ce qui lui assurerait une voie triomphale vers l'apogée du règne. » (f. 33)

51. - «Un nouveau rapport de M. Hermann von Rittgen, l'attaché de presse allemand », note $n^{\circ} 30$ (3295) du 22 mai 1937 de la DGP, ANIC, DGP, vol. 17/1937, f. 37-38.

52. - Corneliu Zelea Codreanu, Insemnarii de la Jilava [Notes de Jilava], Bucarest, Ed. Majadahonda, p. 88. 
l'inculpé souhaitait « espionner et utiliser le moment de carence éventuelle de l'autorité de l'État dans un but insurrectionnel », mais aussi « que durant l'année 1935, il est entré en liaison avec une organisation étrangère, dans le but de recevoir des instructions et des aides de toute nature, pour préparer la révolution sociale en Roumanie ${ }^{53}$. Le juge militaire évoquait ainsi un document visiblement adressé à une officine allemande auprès de laquelle un chef nationaliste roumain qui ne signait pas le document ou dont le nom était occulté se présentait à la tête du «Comité central des légionnaires nationaux-socialistes de Roumanie » et promettait « la future alliance économique et politique », la remise à plus tard de la guerre et la victoire en Roumanie, l'année suivante, de la même formation politique ${ }^{54}$. Bien évidemment, dans le contexte de 1935, Codreanu n'avait jamais caché son hostilité à la signature des traités franco-soviétique et soviéto-tchécoslovaque auxquels la Roumanie devait s'intégrer pour laisser passer l'Armée rouge. Mais la suite de l'accusation selon laquelle cette alliance devait « faciliter la réalisation en Roumanie d'un régime étranger ${ }^{55}$ force bien entendu le trait dans le sens d'une subordination que Codreanu n'envisageait pas, même dans le secret de ses concertations avec ses conseillers, et dont les archives allemandes consultées par Armin Heinen ne gardent pas trace. L'accusation se place sur un terrain plus solide avec le reproche de rébellion et d'organisation paramilitaire en vue de saisir le pouvoir ${ }^{56}$, même si l'accusation de guerre civile masque la tactique mixte, combinant légalisme et violence, du mouvement fasciste.

En définitive, Codreanu fut inculpé pour " crime de trahison », « délit d'intrigue contre l'ordre social » et « crime de rébellion ${ }^{57}$. Le verdict tomba dans la nuit du 26 au 27 mai 1938 et reconnut Codreanu coupable de tous les chefs d'accusation, sans aucune circonstance atténuante : le tribunal militaire le condamna à dix ans de travaux forcés, à six ans de dégradation civique, à cinq mille lei d'amende et à deux mille lei de frais de jugement ${ }^{58}$. Durant sa détention, Codreanu demanda la modération à ses partisans, afin d'éviter son assassinat par le roi et la répression de ses troupes - validant ainsi la tactique semi-révolutionnaire et semi-légaliste d'arrivée au pouvoir des mouvements fascistes. Mais ces derniers, pour maintenir la mobilisation qui risquait de faiblir parmi les militants, entretinrent au contraire l'agitation terroriste.

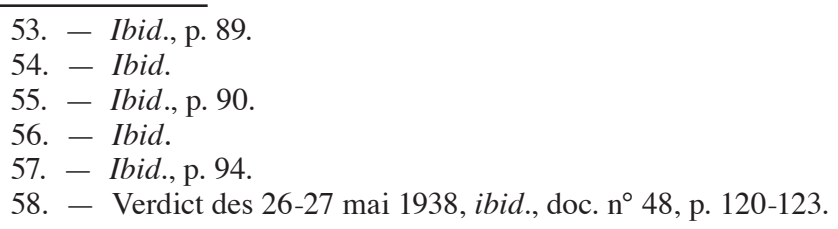


Lors de ses visites prévues à Londres et à Paris, Carol voulait prendre la mesure de la volonté de résistance des Occidentaux au moment du premier arbitrage de Vienne du 2 novembre 1938, qui rendait à la Hongrie la frange méridionale de la Slovaquie et de la Ruthénie tchécoslovaques au nord de la Roumanie. Il avait besoin de donner une image de stabilité et de fidélité de son pays, afin d'inciter les alliés à le protéger en cas de prétentions révisionnistes. L'agitation légionnaire qui s'y préparait était donc particulièrement mal venue pour la diplomatie officielle roumaine, alors que les manifestes d'Alexandru Canacuzino reprenaient les lettres ouvertes de Codreanu au roi de 1936 et de 1937 sur l'hostilité de la Légion envers le système d'alliance roumain et l'appel à une alliance avec l'axe Rome-Berlin. À l'intérieur comme à l'extérieur, les légionnaires contrevenaient à la politique du roi encore alignée sur les alliés traditionnels et sur la gestion autoritaire du pays. Les attentats correspondirent au voyage du roi, commencé le 15 novembre. La liste de la police pour le mois de novembre fait état de dix-huit attentats de gravités diverses ${ }^{59}$ et de publications qui constituaient un camouflet pour le roi 60 .

Or, malgré l'accueil reçu à Londres et à Paris et les discours britanniques sur la nécessité de rester armés, Carol II n'obtint pas d'assurances fermes quant à la sécurité de son pays et chargea le conservateur Constantin Argetoianu et des membres de la famille de Hohenzollern de lui assurer une entrevue informelle avec Hitler. " Des espérances commençaient à poindre », en conclut le chef légionnaire Constantin Papanace à l'époque, même si des inquiétudes subsistaient en cas de défense trop avérée de la part du Führer ${ }^{61}$. Selon certains, Hitler aurait demandé à Carol II d'amener la Garde de fer au pouvoir, ce qui revenait à mettre fin à la dictature royale. Selon d'autres, c'est précisément parce que Hitler n'avait pas évoqué la question que le roi se permit de faire assassiner le Căpitan - lors d'un simulacre de tentative d'évasion dans la nuit du 29 novembre $1938^{62}$-, ce qui fut confirmé par la suite. Les relations germano-roumaines se ressentirent de cet événement et les dignitaires allemands rendirent les décorations attribuées par le roi $\mathrm{Carol}^{63}$. Deux mois plus tard, le ministre des Affaires étrangères

59. - «Exposé chronologique des événements et faits les plus importants survenus en lien avec le Mouvement légionnaire, du 16 février 1938 jusqu'au 20 mars 1939 », rapport de la DGP de mars 1939, ibid., doc. n¹46, p. 240-245, ici p. 242-243.

60. - Curierul legionar, ${ }^{\circ} 1$ du 20 novembre 1938, cité dans Scurtu et al., op. cit., doc. $\mathrm{n}^{\circ} 107$, p. 187-195.

61. - Constantin Papanace, Fără Căpitan. Conducerea în a doua prigoană [Sans le Căpitan. Le commandement durant la deuxième répression], Bucarest, Ed. Elisavaros, 1997.

62. - Communiqué du Parquet militaire du Deuxième corps d'armée du 30 novembre 1938, Scurtu et al., op. cit., doc. n¹08, p. 196.

63. - Scurtu et al., ibid., chronologie, p. 32. 
Grigore Gafencu s'ouvrit à un fonctionnaire de l'ambassade allemande dans le sens «que le Führer continu[ait] à avoir un sérieux préjugé contre la Roumanie à la suite des événements du 30 novembre, considérés comme étant dans un "rapport tragique" avec la visite du roi »64. Hitler avait donc l'impression que son attitude avait contribué à l'élimination de Codreanu, ce dont le roi, à travers Gafencu, voulut le détromper en le persuadant que «durant sa visite en Allemagne, il ne connaissait pas la situation grave de Roumanie », donc « dans ses conversations avec Hitler et le feldmaréchal Göring, il n'a pas pu discuter des mesures nécessaires qui devaient être prises dans le temps le plus court contre les dirigeants de la Garde de fer... Le gouvernement a cru qu'il se trouvait devant l'alternative de prendre les mesures les plus drastiques ou d'être lui-même écarté » ${ }^{65}$. Cette note confirme la thèse du silence sur la question légionnaire dans les discussions de Berchtesgaden.

\section{Conclusion}

Les relations entre la Garde de fer d'une part et le NSDAP et l'État allemand d'autre part semblent plutôt ténues, pour les raisons instrumentales que j'ai évoquées et qui faisaient désirer à Hitler un regroupement des droites roumaines entre ses mains, ce à quoi Codreanu, en véritable chef fasciste, ne pouvait pas consentir. Cela s'est confirmé après l'Arbitrage de Vienne d'août 1940 accordant la Transylvanie du Nord à la Hongrie : certains dirigeants historiques du Mouvement légionnaire comme Corneliu Georgescu ou Radu Mironovici étaient prêts à risquer une guerre contre l'Allemagne ${ }^{66}$. De même, les légionnaires furent réprimés par le général Antonescu avec l'approbation de Hitler en janvier 1941 lors de leur rébellion contre le militaire réactionnaire, qui avait partagé difficilement le pouvoir avec ces jeunes révolutionnaires violents qui ne pouvaient pas non plus convenir à Hitler. Ce dernier avait besoin de tranquillité à l'intérieur de la Roumanie pour l'aligner sur le front de l'Est.

Mais après la répression, c'est en Allemagne que les légionnaires les plus compromis se sont réfugiés ; et quand l'opération Barbarossa fut lancée, les légionnaires se sont bien battus sur le front de l'Est ; enfin, c'est à Vienne que Horia Sima forma, après la chute d'Antonescu en août 1944, un gouvernement pro-allemand en exil. Cela confirme, à mon avis, la collusion de temps de crise liée à la parenté idéologique. $\mathrm{Si}$ elle ne remplace pas les relations de la Realpolitik entre les États, elle

64. - Rapport politique de Stelzer au ministère allemand des Affaires étrangères du 19 janvier 1939, cité dans Scurtu et al., op. cit., doc. n¹27, p. 220-221, ici p. 221.

65. - Ibid.

66. - Rapport d'information de la DGP du 31 août 1940, ibid., doc. n²36, p. 368. 
surgit chaque fois que les régimes, les mouvements ou les personnalités fascistes sont en danger. Et le danger était inhérent au fascisme, depuis que Mussolini avait adopté le vivere pericolosamente nietzschéen.

Je propose de terminer par deux citations, de Codreanu et de Hitler. La défense de Codreanu lors de son procès en 1938 s'ouvrit sur une affirmation du caractère populiste et palingénésique - de renaissance nationale et sociale, selon l'expression popularisée par Roger Griffin dans sa définition du fascisme 67 - de son mouvement :

L'accusation que l'on porte contre moi aujourd'hui par l'ordonnance définitive, je l'ai déjà rencontrée, et elle est, semble-t-il, toujours liée à ces mouvements de renaissance morale et nationale sur lesquels ceux qui les haïssent jettent, avec toutes les invectives, toutes les accusations du monde 68 .

Évidemment, en se lançant dans un historique des accusations de collusion avec Mussolini et surtout avec Hitler, Codreanu comptait surtout désamorcer les accusations de «trahison » et d'un mouvement « stipendié ». J'insiste ici sur la phrase « toujours liée à ces mouvements ", avec une valeur métalinguistique pour moi, car le lien ne s'appuie pas sur les relations entre mouvements, mais plutôt sur l'idéologie palingénésique qui lie les mouvements. Ainsi Codreanu lance-til une menace subliminale à la cour, rappelant que, s'il n'était pas le mercenaire de Hitler, Hitler pourrait un jour se rappeler comment le régime de Carol avait traité un mouvement similaire.

Deux ans plus tard, bien qu'il donnât carte blanche à Antonescu pour réprimer la Légion assimilée à la SA turbulente de la Nuit des longs couteaux, Hitler avertit Antonescu de ne pas rompre avec le soutien populaire que les légionnaires représentaient :

D'un point de vue historique, nous pouvons dire, sur la base d'une vaste expérience, que tout régime doit s'appuyer sur le peuple. L'histoire a montré que là où manque ce fondement et où le régime s'appuie seulement sur la force, dans le meilleur des cas il ne survit pas à la personnalité exceptionnelle qui l'a établi ${ }^{69}$.

67. - Roger Griffin, The Nature of Fascism, op.cit., p. 26 : «a palingenetic form of populist nationalism ». p. 99.

68. - Défense de Codreanu du 23 mai 1938, Scurtu et al., op. cit., p. 98-112, ici

69. - Entrevue d'Obersalzburg du 14 janvier 1941, Antonescu-Hitler, Corespondenţă şi întîlniri inedite (1940-1944) [Antonescu-Hitler, correspondance et rencontres inédites (1940-1944)], édités par Ion Ardeleanu, Vasile Arimia et Ştefan Lache, Bucarest, Ed. Cozia, 1991, vol. I, p. 67. 
Ainsi, si Hitler profitait de la répression du mouvement fasciste en Roumanie pour assurer la sécurité sur les arrières de Barbarossa, sa pensée politique à long terme le ramenait à une légitimation populiste, anti-conservatrice et transnationale du régime, cette " vaste expérience » historique qu'il invoque face à Antonescu du haut de sa puissance qu'il espérait millénaire. 
